\title{
Extensión e inversión tectónica alpinas en el área de Sariego. Control ejercido por la estructura varisca subyacente (Asturias, norte de España)
}

\section{Alpine extension and inversion tectonics in the Sariego area. Control exerted by the underlying Variscan structure (Asturias, northern Spain)}

\author{
J. L. Alonso ${ }^{1}$, E. Barrón ${ }^{2}$, B. González-Fernández \\ E. Menéndez Casares ${ }^{3}$ y J. C. García-Ramos ${ }^{4}$ \\ ${ }^{1}$ Departamento de Geología, Universidad de Oviedo, C/ Jesús Arias de Velasco s/n, 33005 Oviedo, Asturias, España \\ (e-mail: jlalonso@geol.uniovi.es) \\ ${ }^{2}$ Museo Geominero, Instituto Geológico y Minero de España, C/ Ríos Rosas, 23,28003, Madrid, España \\ (e-mail: e.barron@igme.es) \\ ${ }^{3}$ Departamento de Explotación y Prospección de Minas, Universidad de Oviedo, C/Independencia 13, 33004, Oviedo, España \\ (e-mails:mbeagf@uniovi.es ecasares@uniovi.es) \\ ${ }^{4}$ Museo Jurásico de Asturias (muja), Colunga, Asturias, España \\ (e-mail: jcgramos.muja@gmail.com)
}

Resumen: Dos de las fallas mayores de rumbo E-O, que afectan a la cuenca pérmico-mesozoica asturiana (Fallas de Llanera y Careses), pasan por el área de Sariego. Un afloramiento de gran interés pedagógico situado en el talud de la autovía del Cantábrico, cerca de la localidad de Lamasanti, ilustra muy bien el significado de la Falla de Llanera. Dicha falla jugó como falla normal durante el Jurásico Superior y parte más baja del Cretácico Inferior y como falla inversa durante la orogenia Alpina. Su desplazamiento normal fue mayor que el inverso, observándose el punto nulo en el afloramiento mencionado. En ese mismo afloramiento se ha datado con polen la base de la secuencia post-rift del bloque inferior de la falla, obteniéndose una edad Barremiense, siendo la primera vez que se registra este piso en la cuenca mesozoica asturiana. Respecto a la Falla de Careses, se muestra en su sector oriental como una falla normal invertida. Sin embargo, en su sector occidental es una falla inversa que puede interpretarse como una falla de atajo de la falla normal mencionada; el juego inverso de esta falla se refleja en el relieve actual dando lugar a un escarpe mucho más notable que el de la Falla de Llanera. Las dos fallas mayores mencionadas se encuentran cortadas por otras de rumbo SO-NE que representan la reactivación de las estructuras variscas subyacentes durante la orogenia alpina, jugando probablemente en transpresión, como fallas de desgarre con ligero movimiento inverso, generando pliegues subparalelos a las mismas. No obstante, existen evidencias de que estas estructuras variscas se reactivaron previamente como fallas normales, controlando los espesores de la sucesión pérmica. La edad relativa de los diferentes sistemas de fallas durante el acortamiento alpino es la siguiente: primero actuaron las fallas de Llanera y Careses, despúes las de rumbo NE-SO y por último actuó la Falla de Ventaniella, que trunca a todas ellas.

Palabras clave: inversión tectónica, punto nulo, estructuras de basamento reactivadas, Barremiense, palinomorfos, Cuenca Asturiana. 


\begin{abstract}
Two major structures involving the Permian-Mesozoic Asturian Basin (Llanera and Careses faults) are analysed in the Sariego area. The Llanera Fault played as a syn-sedimentary normal fault during the Upper Jurassic-lowermost Cretaceous and was inverted in Cenozoic times. Its reverse displacement was lower than the previous normal displacement and the null point is exposed in an illustrative outcrop located near the Lamasanti village, in the lateral talus of the Cantabrian motorway (A64-E70). In this outcrop, sampling was carried out at the base of postrift succession, in order to obtain palynomorphs, which have provided a Barremian age. This stage has not been recorded in the Asturian Basin so far. The Careses Fault is mainly a reverse fault and is responsible for the major relief of the study area; however, the eastern part of this fault can be recognized as an inverted normal fault, whereas its western part can be interpreted as a short cut thrust of that normal fault. Both, the Llanera and Careses faults are truncated by several SWNE trending faults, which mean the reactivation of buried variscan structures during the Alpine deformation. The map pattern of these SW-NE trending faults implies an oblique displacement, composed of strike and vertical slip; however, these faults played previously as syn-rift extensional faults in Permian times, as recorded by thickness changes in the Permian succession.
\end{abstract}

Keywords: tectonic inversion, null point, inherited basement structures, Barremian, palynomorphs, Asturian Basin.

Desde el punto de vista geográfico, el área objeto de este trabajo se sitúa en la cabecera del río Nora, ocupando aproximadamente la mitad suroriental del municipio de Sariego, en Asturias. Su situación geológica puede verse en la figura 1A. La sucesión estratigráfica de este área forma parte de lo que se ha denominado cuenca permo-mesozoica asturiana, la cual representa un fragmento de la Cuenca Vasco-Cantábrica, de la que actualmente se encuentra desconectada por procesos erosivos asociados al levantamiento de la cordillera Cántabro-pirenaica.

La historia geológica post-varisca de la región comienza con un primer episodio extensional que dio lugar al desarrollo de las cuencas continentales permo-triásicas (Suárez Rodríguez, 1988; Espina et al., 2004), dispuestas en discordancia angular sobre el basamento varisco. Le sucede una cuenca marina del Jurásico Inferior-Medio, atribuida a subsidencia térmica. Una segunda etapa extensional tuvo lugar durante el Jurásico Superior-Cretácico Inferior (Lepvrier y Martínez-García, 1990; Uzkeda et al., 2016), en relación con la apertura del Golfo de Vizcaya, dando lugar a la formación de las principales cuencas mesozoicas, que permanecen estables a partir del Barremiense hasta la inversión tectónica que se produjo durante el Cenozoico y dio lugar a la Cordillera Cantábrica (Alonso et al., 1996). La dirección de la extensión se ha estimado que ocurrió en dirección N-S (Uzkeda et al., 2016), mientras la dirección del acortamiento alpino es generalmente NNO-SSE, aunque oscila entre NO-SE y NE-SO (Lepvrier y Martínez-García, 1990; Espina et al., 1996, Quintana et al., 2006; Uzkeda et al, 2016).

En la zona estudiada están presentes dos de las principales fallas que involucran a la sucesión permo-mesozoica asturiana (fallas de Llanera y Careses) (Fig. 1). Ambas fallas fueron consideradas por Lepvrier y Martínez-García (1990) como cabalgamientos alpinos. Las diferencias estratigráficas entre los dos labios de la Falla de Llanera (Fig. 1A) llevaron a Alonso et al. (1996) a interpretar dicha falla como normal durante el Jurásico Superior, cuyo juego inverso posterior (inversión tectónica) dio lugar a la cuña clástica representada por la cuenca cenozoica de Oviedo (Fig. 1C).

La geología del área de Sariego, que se muestra en un nuevo mapa (Fig. 2A), permite dar mayores precisiones sobre el valor del desplazamiento normal e inverso de la Falla de Llanera, la edad de sus movimientos y sobre el papel de ambas fallas en la creación del relieve actual. Además, el área estudiada presenta un buen desarrollo de fallas de rumbo SO-NE, que pueden relacionarse con la reactivación de las estructuras variscas subyacentes; se discutirá el papel de estas fallas en el desarrollo de la cuenca pérmica y su relación espacial y temporal con las fallas E-O antes mencionadas. Además, se aporta una nueva datación 

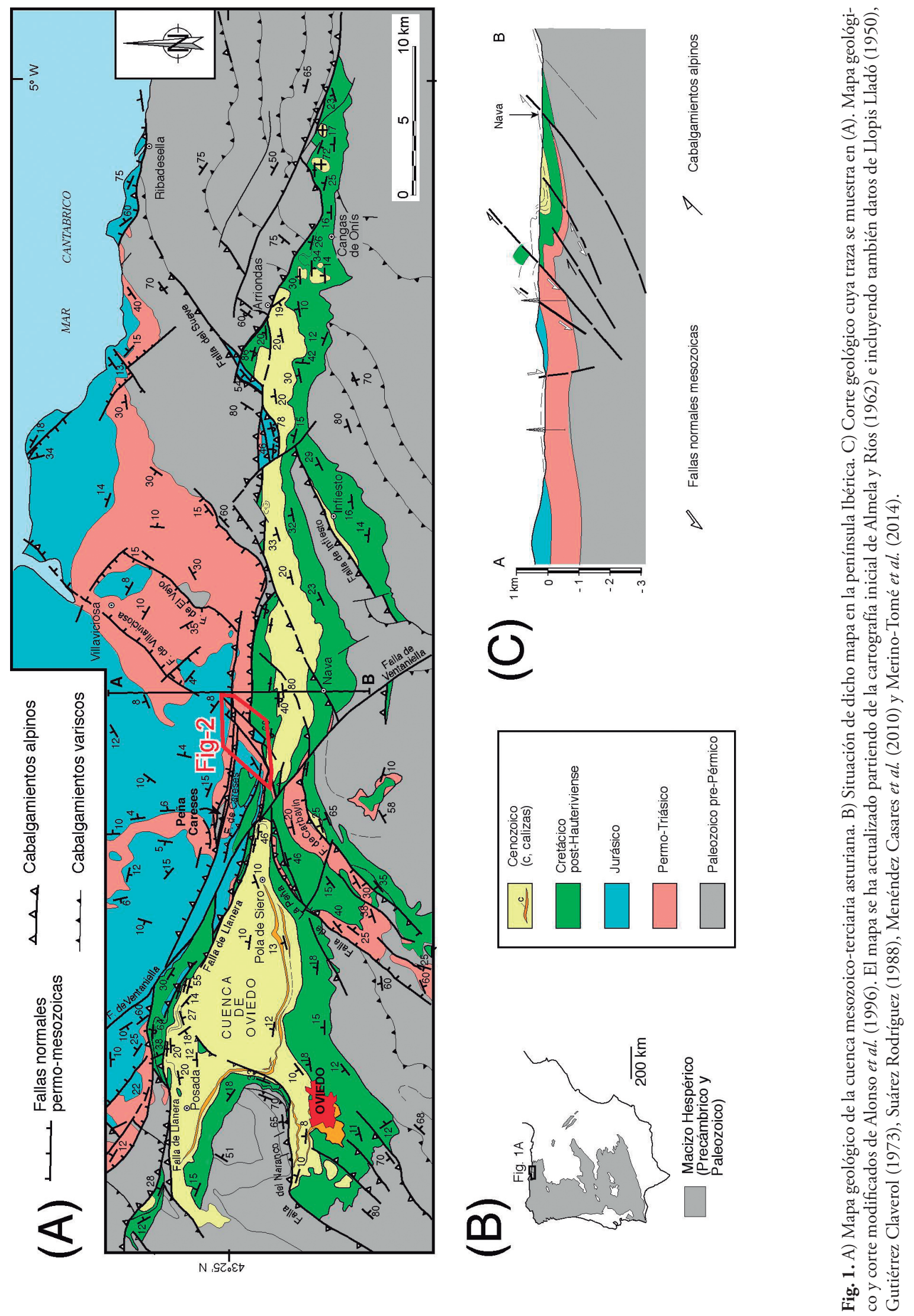


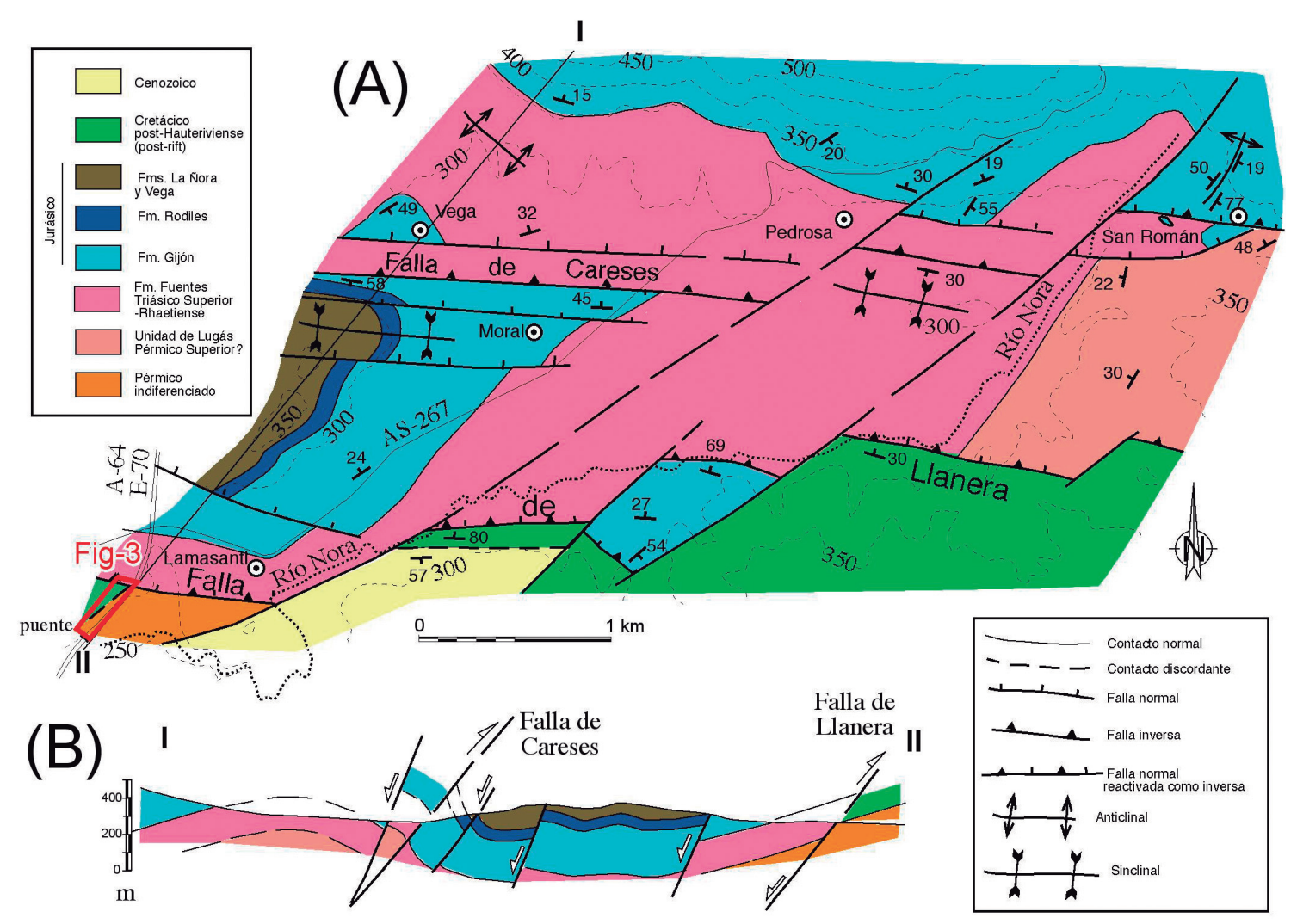

Fig. 2. A): Mapa geológico del sector suroriental del concejo de Sariego. Situación en la Fig. 1. B) Corte geológico cuya traza se muestra en dicho mapa.

(Barremiense) de la base de la sucesión post-rift inmediatamente al sur de la Falla de Llanera, que proporciona una edad cretácica más antigua que la hasta ahora conocida.

\section{Estratigrafía}

La sucesión estratigráfica presente en el área estudiada abarca desde el Pérmico al Cenozoico, con una importante interrupción sedimentaria en el bloque sur de la Falla de Llanera, debido a la actividad sinsedimentaria de dicha falla durante su juego normal, con erosión del bloque inferior, situado al sur (Fig.1A y C).

Los materiales más antiguos presentes en el área estudiada son de edad pérmica y aparecen al sur de la Falla de Llanera en el extremo suroccidental del mapa de la Fig. 2A, y en el extremo oriental de dicho mapa al norte de dicha falla. Se trata de una sucesión de limolitas, lutitas y areniscas calcáreas de tonali- dades rojizas, que incluyen a menudo nódulos carbonatados de aspecto amigdaloide. El afloramiento suroccidental incluye un conglomerado de cantos volcánicos, justo al norte del puente sobre la autovía indicado en dicho mapa; dicho conglomerado está representado en la Fig. 3A. Esta unidad tiene rasgos comunes con sucesiones de áreas próximas, en donde ha sido datada como pérmica (Wagner y Martínez García, 1982). Al norte de la Falla de Llanera, la unidad basal también está constituida por lutitas y areniscas calcáreas con nódulos carbonatados, aunque no presenta participación volcánica; puede considerarse correlacionable con la denominada Unidad de Lugás por Suarez Rodríguez (1988) en la cuenca de Villaviciosa, situada al $\mathrm{N}$ del área estudiada. Por encima de la unidad anterior se sitúa una sucesión de arcillas y lutitas rojas de aspecto masivo con alguna intercalación fina de limolitas y areniscas, así como de yesos, cuya proporción aumenta hacia la parte superior. Esta unidad, denominada Formación Fuentes, ocupa la mayor parte del terreno llano que caracteriza la parte oriental del concejo de Sariego (Fig. 

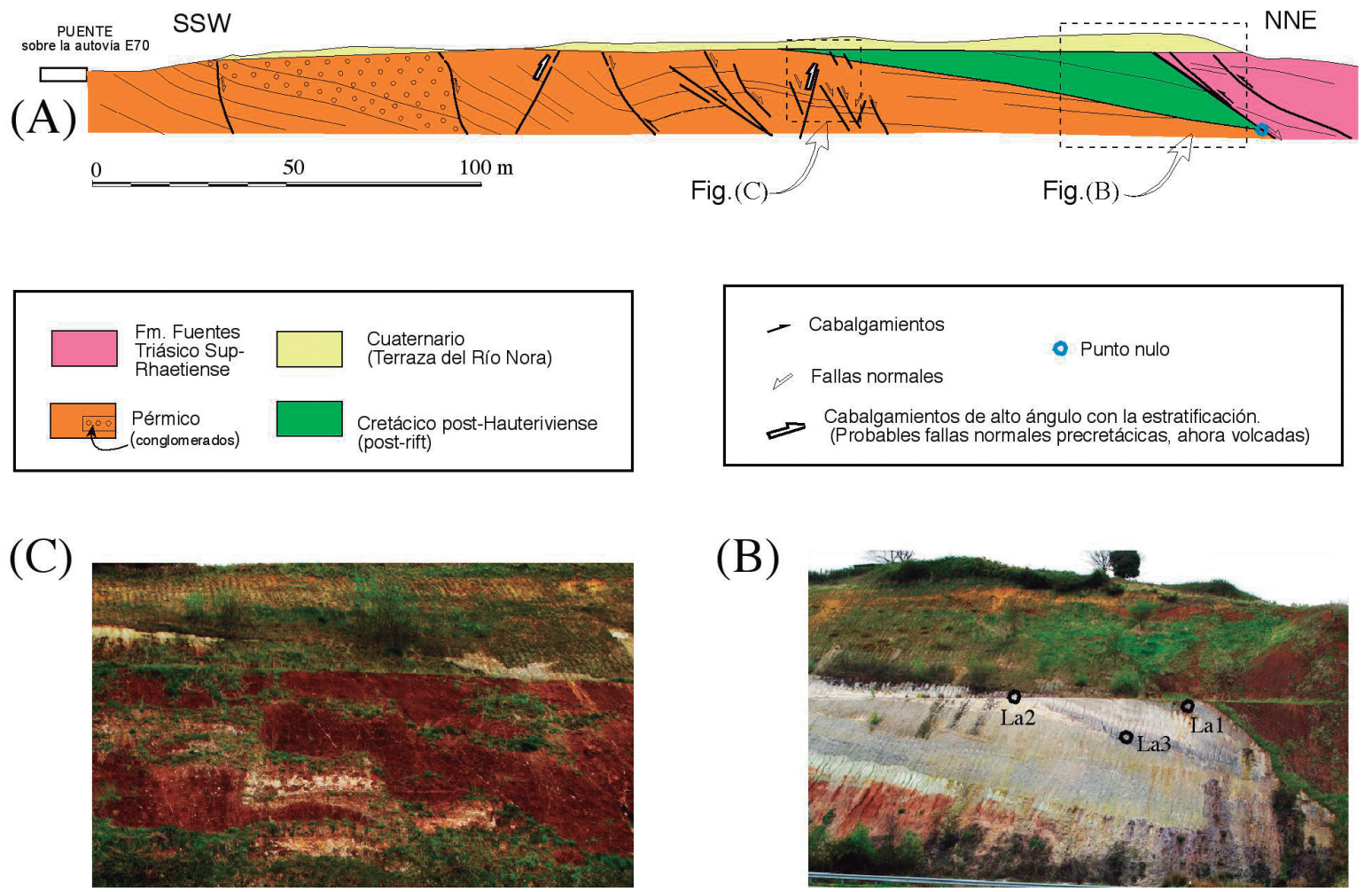

Fig. 3. A) Esquema geológico del talud de la autovía del Cantábrico en el desvío para la localidad de Lamasanti. Situación en Fig. 2. B) y C) Fotografías de los sectores indicados en (A). En B pueden verse los niveles oscuros carbonosos donde se tomaron las muestras (La1, La2 y La3) que proporcionaron el polen.

2A); sus características litológicas presentan similitud con las facies Keuper (Triásico Superior) y su edad, al menos en parte, es Rhaetiense (Martínez García et al., 1998; Barrón et al., 2002). El espesor de esta formación, aunque variable, es de unos $160 \mathrm{~m}$.

Sobre la Formación Fuentes se dispone la Formación Gijón, que representa el Triásico Superior y la parte basal del Jurásico Inferior (Suárez Vega, 1974; Valenzuela et al., 1986). Consiste en una sucesión litoral y marina de dolomías, margas y margocalizas grises oscuras con algunos niveles ricos en lamelibranquios y gasterópodos. Hacia la base también aparecen brechas carbonatadas. A esta unidad le sucede la Formación Rodiles, de edad Jurásico Inferior-Medio y también de origen marino; está constituida por una alternancia rítmica de calizas y margas. En el área estudiada, el Jurásico Superior está representado por las formaciones La Nora y Vega, de carácter continental, que aparecen en el sector occidental del mapa (Fig. 2A); estas dos formaciones están constituidas por conglomerados silíceos, areniscas y lutitas de carácter fluvial. En el área estudiada, el espesor total de estas cuatro formaciones jurásicas es de unos $400 \mathrm{~m}$ y solamente están presentes en el bloque norte de la Falla de Llanera (Fig. 2 A y B).

La serie cretácica más antigua del área estudiada aparece en el extremo suroccidental del mapa (Fig. 2A), en ligera discordancia angular sobre la sucesión pérmica (Fig. 3A) y representa la base de la secuencia post-rift. Se trata de conglomerados de cantos cuarcíticos, arenas blancas y algunos niveles de lutitas con restos carbonosos. La edad precisa de la base de la sucesión cretácica en este sector era desconocida, por lo que se tomaron muestras de los materiales carbonosos (muestras La1, La2 y La3 en Fig. 3B) con el fin de tratar de llevar a cabo una datación con polen y esporas. El resultado fue positivo y la lista de especies se muestra en la Tabla 1, proporcionando una edad Barremiense, siendo la primera vez que este piso es datado en Asturias; dicha edad indica que estos materiales probablemente pertenecen a la Formación Peñaferruz, que hasta ahora se consideraba exclusiva del sector situado al norte de la Falla de Llanera (González-Fernández et al., 2004). Además, 
esta edad Barremiense para la base de la secuencia post-rift es la más antigua encontrada al sur de la Falla de Llanera, en donde son habitualmente las formaciones albienses (formaciones Pola de Siero o Ullaga) las que se apoyan sobre el basamento varisco o sobre el Pérmico.

Por encima de la Fm. Peñaferruz se encuentra una serie atribuida a la Formación Antromero, de edad Aptiense (Schroeder y Wienands, 1966), constituida por areniscas, lutitas y niveles carbonosos con intercalaciones de calizas con abundantes ostreidos y gasterópodos, que afloran inmediatamente al oeste de la zona estudiada. La serie cretácica continúa con la Formación Pola de Siero, formada por un tramo inferior de conglomerados silíceos con intercalaciones de arcillitas rojas y otro superior de areniscas con niveles de arcillitas, limolitas y materia carbonosa. Finalmente, el Cretácico más moderno aflorante en la zona está representado por la Formación Ullaga, de edad Albiense, constituida fundamentalmente por calizas bioclásticas, con tramos de arcillitas, limolitas y areniscas con materia carbonosa y ámbar (González-Fernández et al., 2004). Estas dos formaciones están presentes en el área estudiada al sur de la Falla de Llanera, al este del río Nora (Fig. 2A).
Respecto al Cenozoico representado en la Fig. 2A, que forma parte de la denominada Cuenca de Oviedo (Fig. 1A), está constituido principalmente por conglomerados calcáreos y areniscas, que forman parte de una orla de abanicos aluviales coalescentes de baja eficacia de transporte y alta pendiente adosados al borde activo de la cuenca, situado al norte y constituido en su mayor parte por rocas cretácicas (Fig. 1A) (García-Ramos y Gutiérrez Claverol, 1995). La sucesión de la cuenca de Oviedo es de edad paleógena, probablemente Eoceno Superior-Oligoceno Inferior (Truyols et al., 1991).

\section{Estudio palinológico de la sucesión cretácica de Lamasanti}

Se han preparado con el método palinológico clásico (Traverse, 2007), tres muestras del afloramiento de Lamasanti, una de ellas (Lamasanti 1), no ha presentado contenido palinológico. Las otras dos han proporcionado cantidades representativas (Tabla 1) aunque, por lo general, en mal estado de conservación, de miosporas (esporas de criptógamas y granos de polen). Algunas de las especies se ilustran en la Fig. 4. No se han hallado dinoquistes, acritarcos,

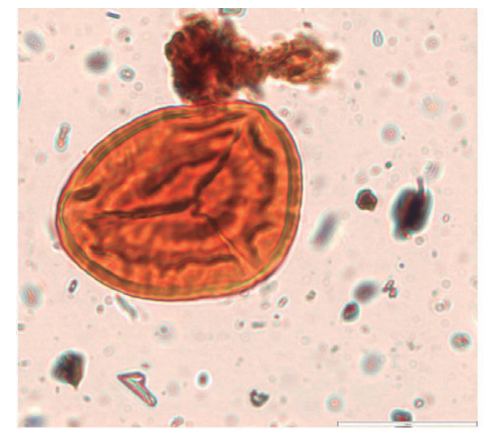

(a)

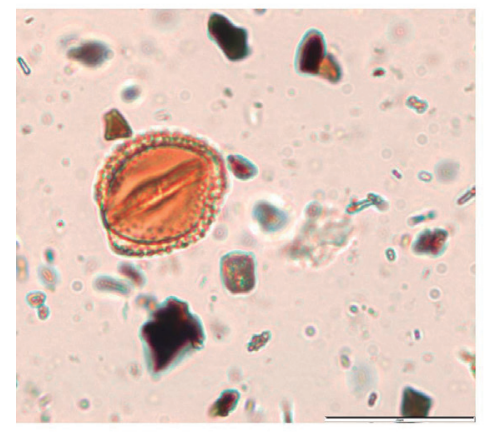

(c)

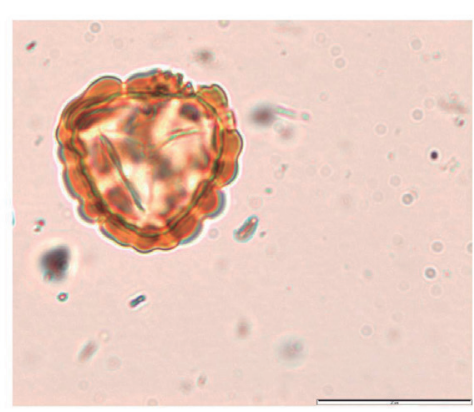

(b)

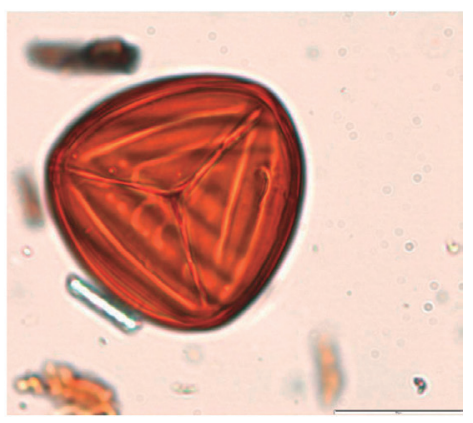

(d)
Fig. 4. Fotografías de algunos de los palinomorfos del yacimiento de Lamasanti. a) Staplinisporites telatus. b) Leptolepidites macroverrucosus. c) Clavatipollenites. (d): Cicatricosisporites hughesii. La escala del ángulo inferior derecho de las fotos es de 20 micras. 
Lamasanti $2 \quad \% \quad$ Lamasanti $3 \quad \%$

\section{Esporas}

Appendicisporites bilateralis Singh 1971

Biretisporites potoniaei Delcourt \& Sprumont 1955

Camerosporites sp.

Cicatricosisporites apicanalis Phillips \& Felix 1971

Cicatricosisporites apitereus Phillips \& Felix 1971

Cicatricosisporites hughesii Dettman 1963

Cicatricosisporites cf. subrotondus Brenner 1963

Cicatricosisporites venustus Deák 1963

Cicatricosisporites spp.

Cicatricososporites auritus Singh 1971

Cingutriletes sp.

Cyatbidites australis Couper 1953

Cyathidites minor Couper 1953

Deltoidospora sp.

Densoisporites velatus Weyland \& Krieger 1953

Distaltriangulisporitessp.

Echinatisporis sp.

Gleicheniidites senonicus Ross 1949

Laevigatosporites sp.

Leptolepidites macroverrucosusSchulz 1967

Lycopodiumsporites sp.

Maculatisporites sp.

Neoraistrickia truncata (Cookson 1953) Potonié 1956

Osmundaciditessp.

Patellasporites tavaredensis Groot \& Groot 1962

Rotverrusporites major (Couper 1953) Fensome 1983

Staplinisporites caminus (Balme 1957) Pocock 1962 emend. Fensome 1983

Trachysporites sp.

Todisporites major Couper 1958

Esporas trilete indeterminables

\begin{tabular}{|c|c|c|c|}
\hline 2 & 0,43 & 0 & 0 \\
\hline 1 & 0,22 & 0 & 0 \\
\hline 0 & 0 & 1 & 0,23 \\
\hline 1 & 0,22 & 0 & 0 \\
\hline 1 & 0,22 & 0 & 0 \\
\hline 1 & 0,22 & 0 & 0 \\
\hline 1 & 0,22 & 0 & 0 \\
\hline 1 & 0,22 & 0 & 0 \\
\hline 31 & 6,75 & 9 & 2,09 \\
\hline 1 & 0,22 & 0 & 0 \\
\hline 0 & 0 & 1 & 0,23 \\
\hline 0 & 0 & 2 & 0,46 \\
\hline 7 & 1,53 & 0 & 0 \\
\hline 13 & 2,83 & 15 & 3,48 \\
\hline 1 & 0,22 & 0 & 0 \\
\hline 1 & 0,22 & 0 & 0 \\
\hline 1 & 0,22 & 0 & 0 \\
\hline 0 & 0 & 1 & 0,23 \\
\hline 1 & 0,22 & 1 & 0,23 \\
\hline 1 & 0,22 & 0 & 0 \\
\hline 1 & 0,22 & 0 & 0 \\
\hline 1 & 0,22 & 0 & 0 \\
\hline 4 & 0,87 & 0 & 0 \\
\hline 1 & 0,22 & 3 & 0,7 \\
\hline 2 & 0,43 & 1 & 0,23 \\
\hline 2 & 0,43 & 0 & 0 \\
\hline 0 & 0 & 1 & 0,23 \\
\hline 1 & 0,22 & 0 & 0 \\
\hline 1 & 0,22 & 1 & 0,23 \\
\hline 5 & 1,09 & 0 & 0 \\
\hline
\end{tabular}

Granos de polen (gimnospermas)

Alisporites bilateralis Rouse 1959

Araucariacites australis Cookson 1947

Callialasporites dampieri Dev 1961

Cedripites sp.

Classopollis spp.

Cerebropollenites macroverrucosus (Thiergart 1949) Schulz 1967

Cycadopites spp.

Eucommiidites minor Groot \& Penny 1960

Eucommiidites troedsonii Erdtman 1948

Exesipollenites tumulus Balme 1957

Inaperturopollenites dubius (Potonié \& Venitz 1932) Thompson \& Pflug 1953

Inaperturopollenites spp.

Monosulcites chaloneri Brenner 1963

Monosulcites minimus Cookson 1947 ex Couper 1953

Monosulcites sp.

$\begin{array}{cccc}4 & 0,87 & 7 & 1,63 \\ 1 & 0,22 & 6 & 1,39 \\ 0 & 0 & 1 & 0,23 \\ 1 & 0,22 & 0 & 0 \\ 31 & 6,75 & 89 & 20,65 \\ 2 & 0,43 & 2 & 0,46 \\ 10 & 2,18 & 6 & 1,39 \\ 0 & 0 & 1 & 0,23 \\ 2 & 0,43 & 0 & 0 \\ 4 & 0,87 & 10 & 2,32 \\ 290 & 63,19 & 192 & 44,55 \\ 11 & 2,39 & 8 & 1,87 \\ 4 & 0,87 & 7 & 1,63 \\ 1 & 0,22 & 7 & 1,63 \\ 0 & 0 & 4 & 0,93\end{array}$




\begin{tabular}{|c|c|c|c|c|}
\hline & Lamasanti 2 & $\%$ & Lamasanti 3 & $\%$ \\
\hline \multicolumn{5}{|l|}{ Granos de polen (gimnospermas) } \\
\hline Pinuspollenites sp. & 0 & 0 & 2 & 0,46 \\
\hline Spheripollenites sp. & 3 & 0,65 & 2 & 0,46 \\
\hline Vitreisporites pallidus (Reissinger 1950) Nilsson 1958 & 1 & 0,22 & 1 & 0,23 \\
\hline Granos de polen bisacados indeterminables & 4 & 0,87 & 16 & 3,71 \\
\hline \multicolumn{5}{|l|}{ Granos de polen (angiospermas) } \\
\hline Asteropollis trichotomosulcatus (Singh 1971) Singh 1983 & 1 & 0,22 & 7 & 1,63 \\
\hline Clavatipollenites hughesii Couper 1958 & 3 & 0,65 & 9 & 2,09 \\
\hline Clavatipollenites minutus Brenner 1963 & 1 & 0,22 & 10 & 2,32 \\
\hline Pennipollis sp. & 2 & 0,43 & 6 & 1,39 \\
\hline Granos de polen de angiospermas indeterminables & 0 & 0 & 2 & 0,46 \\
\hline TOTAL miosporas & 459 & 100 & 431 & 100 \\
\hline
\end{tabular}

Tabla 1: Palinomorfos identificados en el Cretácico Inferior (Barremiense) de la sección de Lamasanti (Asturias).

ficomas de tasmanáceas, ni otros palinomorfos que pudieran indicar influencia marina.

La muestra con mayor diversidad es Lamasanti 2, que se encuentra sobrerrepresentada por Inaperturopollenites dubius, grano de polen que se relaciona con cupresáceas. En segundo lugar destacan los valores de Cicatricosisporites, género de esporas trilete cicatricosadas que fueron producidas por helechos de la familia Anemiaceae (Fig. 3a); Classopollis, granos de polen circunsulcados procedentes de coníferas de la extinta familia Cheirolepidiaceae; y esporas trilete deltoides y psiladas del género Cyathidites que en la actualidad están presentes en helechos arborescentes de las familias Cyatheaceae, Dicksoniaceae y Dipteridaceae. Por su parte, en Lamasanti 3, I. dubius no tiene tanta representación, aumentando los porcentajes de Classopollis y disminuyendo bruscamente los de esporas, que también descienden en diversidad de taxones.

La presencia de granos de polen de angiospermas indica una edad cretácica para las dos muestras estudiadas. Las angiospermas que aparecen son representantes de formas monosulcadas y reticuladas de los géneros Clavatipollenites y Pennipollis, que se relacionan con plantas herbáceas de la familia Chloranthaceae y de la clase Monocotyledoneae, respectivamente. De forma puntual se ha hallado un grano de polen tricotomosulcado de la especie Asteropollis trichotomosulcatus en Lamasanti 2.

Los registros más antiguos de granos de polen de angiospermas en la Península Ibérica proceden del Valanginiense de Porto da Calada (cuenca Lusitánica, Portugal) (Trincão, 1990), y corresponden a la especie Clavatipollenites hughesii, que también se ha identificado en Lamasanti. A partir de este período, las angiospermas se diversificaron y aumentaron su presencia y peso en los ecosistemas cretácicos. Sus granos de polen llegaron a ser dominantes en las asociaciones polínicas a partir del Albiense superior (Barrón et al., 2015).

Por una parte, la presencia de Cicatricosisporites venustus, Pennipollis sp., Clavatipollenites minutus y de Monosulcites chaloneri indica que la edad de los niveles estudiados no es más antigua que el Barremiense, ya que las cuatro especies citadas se registran por primera vez en el Hemisferio Norte a partir este piso (Peyrot et al., 2007; Villanueva-Amadoz et al., 2010). Por otra, la escasa diversidad de granos de angiospermas junto con la ausencia de polen tricolpado, cuyos primeros registros en la Península Ibérica corresponden al Aptiense inferior de la cuenca Vasco-Cantábrica (Najarro et al., 2011), restringiría la edad de las muestras estudiadas al Barremiense. 
Desde un punto de vista paleoecológico, por la abundancia y diversidad de esporas, la muestra Lamasanti 2 parece indicar un medio más húmedo que Lamasanti 3, que está caracterizado por valores más elevados de granos de polen de gimnospermas. El clima debió ser cálido, de tipo subtropical. En la Península Ibérica hay pocos datos sobre floras barremienses y parece que estas señalan la existencia de climas cálidos con una estación tórrida y otra, que correspondería con el invierno, más húmeda (Diéguez et al., 2010).

\section{Estructura}

El mapa geológico del área de Sariego muestra tres tipos de fallas de rumbo E-O (Fig. 2A y B): normales, inversas y normales reactivadas como inversas, es decir que sufrieron lo que se denomina inversión tectónica. Además, también aparecen fallas de rumbo NE-SO, que exhiben una combinación de movimientos de desgarre y verticales. En primer lugar se van a describir los rasgos estructurales de las dos fallas mayores de rumbo E-O, denominadas de Llanera y Careses.

\section{La Falla de Llanera}

La Falla de Llanera constituye la estructura alpina más importante de Asturias, ya que su juego como falla inversa, con elevación del bloque norte, es responsable de la formación de la cuenca cenozoica de Oviedo (Alonso et al., 1996) (Fig. 1A y C), cuyos sedimentos son el resultado de la erosión del bloque superior de la falla. En las proximidades de la misma aparece una banda con fallas menores y, en algún sector, pliegues apretados vergentes al $S$, denominada Franja Móvil Intermedia (Ramírez del Pozo, 1969), cuyo desarrollo puede atribuirse a la presencia de una zona de cizalla relacionada con dicha falla mayor.

Además, la falla separa dos sectores con importantes diferencias en la sucesión estratigráfica. $\mathrm{Al}$ sur de la misma, las capas del Cretácico Inferior se apoyan directamente sobre el basamento varisco (Almela y Ríos, 1962), excepto en un pequeño sector situado al sur del área estudiada en donde la sucesión cre- tácica se sitúa sobre las formaciones pérmico-triásicas (Figs. 1 A y C). En contraste con la ausencia de Jurásico al sur de la falla, en el bloque norte de la misma la sucesión jurásica presenta unos $400 \mathrm{~m}$ de espesor en el área estudiada (Fig. 2B). No obstante, aunque la sucesión mesozoica sea más completa en el bloque norte, pueden faltar el Jurásico más superior y el Cretácico Inferior más temprano. De hecho, en la sucesión sin-rift al norte de la falla no se han registrado con seguridad edades más modernas que el Kimmeridgiense (Dubar y Mouterde, 1957) pero, dada la complejidad estructural y la mala calidad de los afloramientos en la faja situada inmediatamente al norte de dicha falla, y la no preservación de la parte superior de la misma sucesión en sectores más septentrionales, debido al nivel de erosión, no debe descartarse que dicha sucesión pudiera incluir el Titoniense y parte del Cretácico pre-Barremiense en algún sector. Considerando que, al menos durante el Jurásico Inferior y Medio, la Falla de Llanera no representaba el borde de la cuenca marina, ello implica una etapa de erosión durante el Jurásico Superior o Cretácico Inferior temprano en el bloque sur de la falla, debido al levantamiento de dicho bloque por el juego normal de la misma en ese periodo de tiempo. En el bloque norte, las capas post-rift de edad Barremiense (Formación Peñaferruz), se apoyan sobre la sucesión sin-rift, mientras en el bloque sur la base de la sucesión post-rift es generalmente algo más moderna (Albiense-Aptiense) y se apoya sobre el basamento varisco o sobre la serie permo-triásica (Almela y Ríos, 1962) (Fig. 1 A y C). Resumiendo, la Falla de Llanera muestra un juego inverso alpino, responsable del desarrollo de la cuenca cenozoica de Oviedo, pero las diferencias estratigráficas entre ambos labios de la misma (Fig. 2B) implican un movimiento normal previo, durante el Jurásico Superior-Cretácico Inferior pre-Barremiense.

En el extremo suroccidental del área estudiada (Fig. 2A) se presenta un excelente afloramiento (Fig. 3), de gran interés pedagógico, en donde se observan estos dos movimientos. Una primera nota sobre dicho afloramiento fue dada a conocer por Alonso et al. $(2009 a)$. El afloramiento se presenta en el talud de la autovía del Cantábrico (A64-E70), junto al desvío para la localidad de Lamasanti. En el bloque superior de la falla, situado al norte de esta, afloran materiales detríticos finos (arcillas masivas con algún delgado 
nivel de limolitas) de color rojizo pertenecientes a la Formación Fuentes, que se considera de edad triásica (Rhaetiense). En el bloque inferior de la misma, situado al sur, se reconoce una sucesión detrítica fina de color rojizo o blanco, con costras carbonatadas y participación volcánica, de edad pérmica, sobre la que se dispone una serie de materiales detríticos (arenas con cantos de cuarzo redondeados y arcillas de color grisáceo con pasadas carbonosas) pertenecientes al Cretácico Inferior (Fig. 3B). La falla se inclina $65^{\circ}$ al NNE (el buzamiento en la Fig. 3 es aparente) y las estrías inversas tienen un buzamiento de $54^{\circ}$ hacia el NE. No se han podido observar las estrías relacionadas con el desplazamiento normal. Tanto los materiales del bloque superior como inferior de la falla presentan un suave buzamiento hacia el noroeste, en torno a $25^{\circ}$. Esta falla presenta evidencias de que su desplazamiento inverso durante la inversión tectónica fue menor que el desplazamiento normal, de manera que en su parte inferior aún conserva parte de su desplazamiento inicial como falla normal. Así, en la parte inferior del afloramiento de la Fig. 3A las rocas de edad triásica (Formación Fuentes) del bloque superior de la falla están directamente en contacto con los materiales más antiguos del Pérmico situados en el bloque inferior de la misma, por lo que la falla muestra un movimiento normal. Por otro lado, en la parte superior del afloramiento los materiales triásicos del bloque superior de la falla están en contacto por medio de esta con las rocas cretácicas post-rift situadas en el bloque inferior, indicando que se trata de una falla inversa. El punto en el cual la falla pasa de tener un movimiento normal a inverso se conoce como punto nulo (Williams et al., 1989). En realidad, el punto nulo fue ilustrado con una falla en donde hay sedimentación continua en ambos bloques de falla (Fig. 5) y en el caso de la falla de Llanera hay erosión del bloque levantado, con ausencia de varias unidades estratigráficas en el mismo por debajo de la sucesión post-rift, lo cual es probablemente más realista que la figura de Williams et al. (op. cit.). En todo caso, la falla de Llanera en la Fig. $3 \mathrm{~A}$ se ajusta desde el punto de vista conceptual a la definición original de punto nulo.

La estructura de la sucesión pérmica en el bloque inferior de la Falla de Llanera consiste esencialmente en fallas normales, tanto planares como lístricas (Fig. 3A), la mayoría de ellas inclinadas al norte. Además, aparece un suave pliegue anticlinal sobre una de las fallas lístricas, en la parte media de la Fig. 3A, que puede interpretarse como un anticlinal de rollover. También están presentes tres fallas inversas, dos de las cuales son prácticamente perpendiculares a las capas (Fig. 3A); si rotamos la sucesión cretácica postrift hasta la horizontal, las dos fallas mencionadas se

\section{(A)}

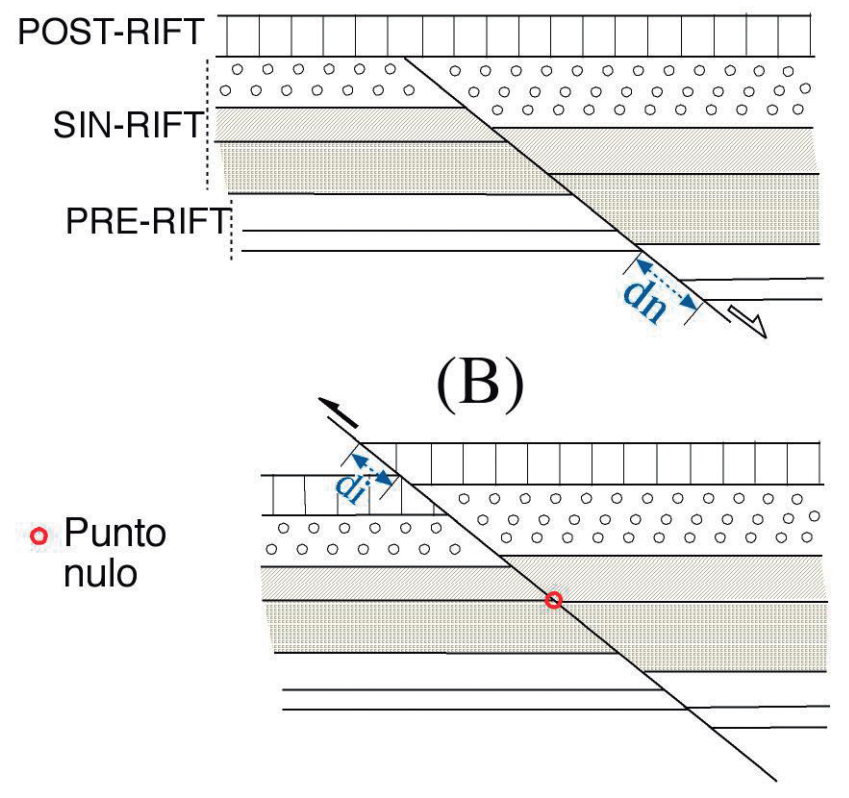

Fig. 5. Esquema mostrando el concepto de punto nulo, el cual separa dos tramos de falla en los cuales esta muestra movimiento normal e inverso respectivamente, cuando existe inversión tectónica. Modificado de Williams et al. (1989). A) Estructura previa a la inversión tectónica. B) Disposición estructural después de dicha inversión. dn: desplazamiento normal; di: desplazamiento inverso. 
muestran como normales, por tanto, se trata probablemente de fallas extensionales volcadas.

\section{La Falla de Careses y otras fallas menores de rumbo E-O}

Al norte de la Falla de Llanera, la falla de rumbo E-O más importante en el área estudiada es la denominada Falla de Careses (Tejerina Lobo y Vargas Alonso, 1980). Se trata de una falla inversa con levantamiento del bloque septentrional, la cual es responsable del afloramiento de calizas carboníferas en la Peña Careses, situada al oeste del área estudiada (Fig. 1A). El mayor escarpe topográfico en el concejo de Sariego parece estar relacionado con dicha falla y no con la de Llanera, a través de la cual no se aprecian diferencias notables en el nivel de cumbres, a pesar de que su separación estratigráfica y desplazamiento inverso son mayores que los de la Falla de Careses (Figs. 1C, 2 y 6). La divisoria de aguas también se encuentra desplazada hacia el norte en este sector, respecto al sector más occidental. Este hecho posiblemente sea debido a que la Falla de Careses actuó en esta área más recientemente que la de Llanera. Al este de la localidad de San Román (Fig. 2), la falla situada al pie del escarpe topográfico de la Fig. 6 se muestra como normal junto a dicha localidad y como inversa con pequeña separación estratigráfica en su prolongación oriental (Figs. 1A y 2A), por lo que en ese sector debe tratarse de una falla normal reactivada como inversa, siendo equiparables los desplazamientos normal e inverso. Esta interpretación es coherente con los datos cartográficos del sector occidental del mapa de la figura $2 \mathrm{~A}$, en donde una falla normal discurre paralelamente a la Falla de Careses unos 100 metros más al norte de ella y con desplazamientos similares; en este sector, la Falla de Careses puede considerarse una falla inversa de atajo (short cut) de dicha falla normal (Fig. 2B), mientras en el sector oriental observamos la propia falla normal invertida (Fig. 2A).

Además de las fallas mayores de Llanera y Careses, las cuales registran inversión tectónica, en el área es-

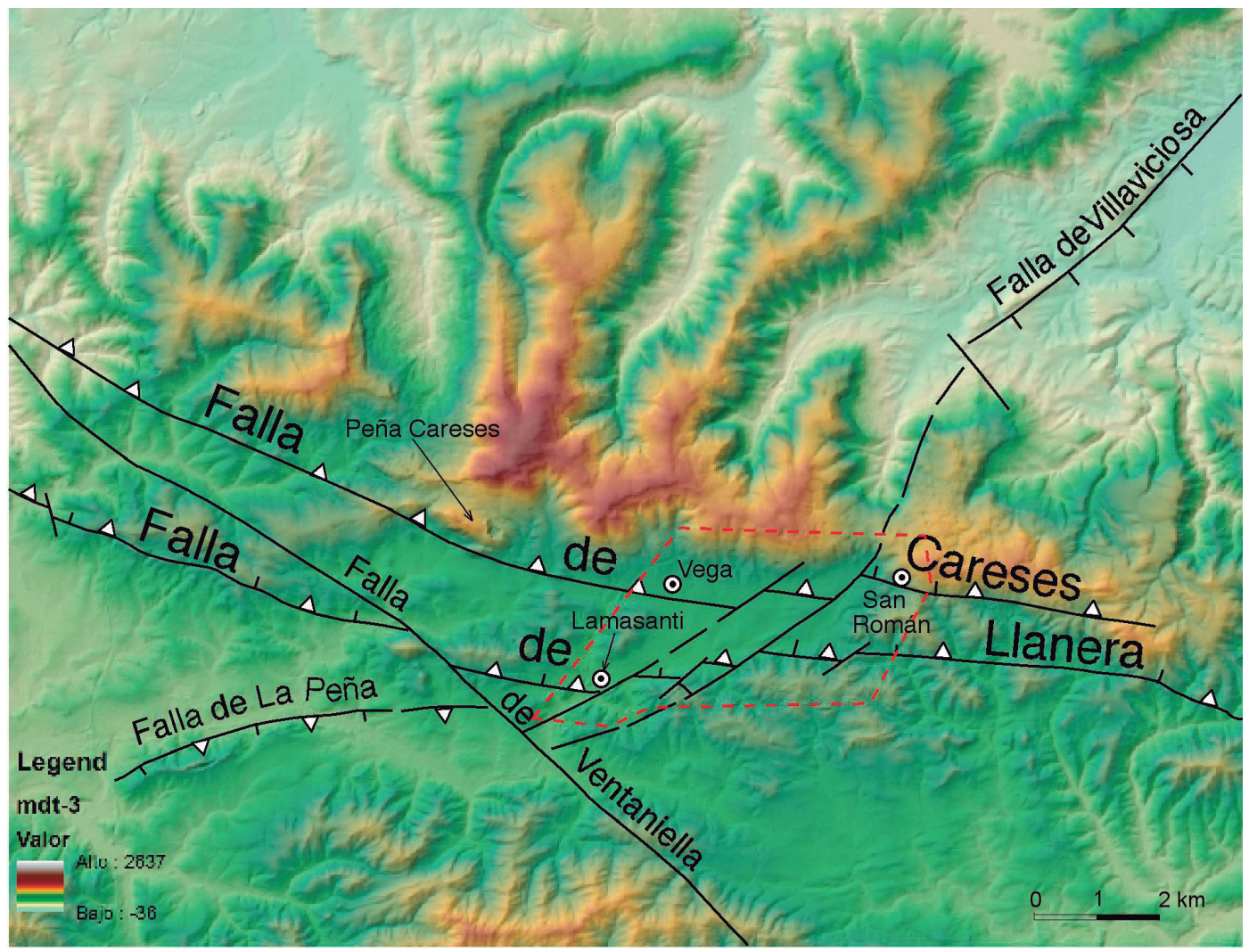

Fig. 6. Modelo digital del terreno mostrando el papel de las fallas de Careses y Llanera en la generación del relieve actual, así como el truncamiento de dichas fallas por las de La Peña-Villaviciosa y Ventaniella. La línea discontinua roja muestra el área ocupada por el mapa de la Fig. 2A. 
tudiada se presentan otras fallas menores de juego normal, con descenso de los bloques septentrionales (Fig. 2 A y B). Estas fallas deben estar en relación con la etapa extensional del Jurásico Superior-Cretácico inferior, ya que involucran a la sucesión jurásica. Fallas extensionales con disposición similar han sido descritas en el área de La Collada-Careses y a veces llevan asociados filones de fluorita; las labores mineras en dicho sector han permitido caracterizar con precisión la geometría de dichas fallas (Jullien, 1974; Tejerina Lobo y Vargas Alonso, 1980).

Las fallas de rumbo SO-NE. Su papel durante la extensión pérmica e inversión alpina. Edad relativa de los diferentes sistemas de fallas durante el acortamiento alpino.

Las fallas SO-NE tienen un rumbo paralelo al trazado de los cabalgamientos y pliegues variscos subyacentes, que afloran alrededor de la cuenca mesozoica (Fig. 1A), por lo que debe tratarse de estructuras variscas reactivadas durante la deformación alpina, tal como ha sido descrito por Pulgar et al. (1999) para otras fallas próximas de la Zona Cantábrica, como el cabalgamiento de Infiesto-Sueve (Fig. 1A). En respuesta a la orientación de los esfuerzos alpinos, que se disponen aproximadamente N-S (Lepvrier y Martínez García, 1990; Espina et al., 1996; Ferrús y Santanach, 1994; Uzkeda et al, 2016), los cabalgamientos variscos de dirección NE-SO, transversales a la cuenca de Oviedo (Infiesto, Carbayín, Naranco) (Fig. 1A), se reactivaron como fallas de deslizamiento flexural en relación con el reapretamiento del Antiforme de Río Color, situado al sur del área estudiada o del Arco Astúrico en su conjunto (Pulgar et al., 1999). Durante el acortamiento alpino, las fallas de dirección NE-SO jugaron mayormente como desgarres levógiros (Uzkeda et al., 2013). En el área estudiada, las fallas NE-SO de Sariego también evidencian un componente vertical, atestiguado por los desplazamientos aparentes opuestos de planos oblicuos cortados por estas fallas; estos planos oblicuos están representados por la falla de Llanera, que muestra un desplazamiento aparente levógiro, y el contacto basal de la Formación Gijón en el norte del mapa, en donde el desplazamiento aparente es dextrógiro y menor que el anterior (Fig. 2A). Además, estas fallas jugaron probablemente en transpresión, generando pliegues subparalelos a las mismas; uno de estos pliegues puede observarse junto a la localidad de San Román (Fig. 2 A). Consideramos, por tanto, que las fallas de traza NE son responsables de que las capas mesozoicas en el entorno de dichas fallas muestren un rumbo varisco (SO-NE), en vez del rumbo E-O que cabría esperar de esfuerzos regionales $\mathrm{N}-\mathrm{S}$ como son los alpinos. De hecho, entre las fallas de Llanera y Careses las capas muestran generalmente una disposición homoclinal con rumbo subparalelo a las fallas NE-SO y buzamiento al NO (Fig. 2 A). Excepcionalmente, las capas muestran a veces un rumbo E-O en la proximidad de las fallas de Llanera y Careses, debido al desarrollo de pliegues relacionados con las mismas (Fig. 2A y B).

Además del papel ejercido por las fallas de rumbo SO-NE durante el acortamiento alpino, también existen evidencias de su actividad durante la extensión pérmica, controlando los espesores de la sucesión de dicha edad. Este control fue reconocido por Sánchez de la Torre et al., (1977) y Suárez Rodríguez (1988) en el área de Villaviciosa-Libardón, situada al NE de la zona de Sariego. En el área objeto de este trabajo también existen evidencias de que las fallas SO-NE representadas en la Fig. 2A separan dos dominios con importantes diferencias de espesor en la sucesión permo-triásica. Mientras inmediatamente al oeste del mapa representado en dicha figura (Sector de Peńa Careses-La Collada) (Fig. 1A) o en el área de La Camocha algo más al norte, el espesor de dicha sucesión no supera los $450 \mathrm{~m}$ (Tejerina Lobo y Vargas Alonso, 1980; Pieren et al., 1995), un sondeo realizado en la localidad de San Román, situada en el extremo oriental de la Fig. 2A, atravesó unos $800 \mathrm{~m}$ de rocas pérmicas y triásicas (Navarro Vázquez et al., 1988). Este gran espesor de sucesión pérmica, situado en el extremo oriental de Sariego, se prolonga al sur de la Falla de Ventaniella en la fosa pérmica situada al sureste de la denominada Falla de La Peña (Figs. 1A) la cual limita por el oeste dicha cuenca pérmica. Dicha falla, desplazada por la Falla de Ventaniella, debe corresponderse en el labio norte de esta última con alguna de las dos fallas de rumbo SO-NE de la Fig. 2A. Al norte del área estudiada debe a su vez enlazar con la Falla de Villaviciosa (Fig. 6), cuya prolongación hacia la costa coincide probablemente con la falla de la ensenada de La Conejera (Uzkeda et al., 2013), también con descenso de su 
labio oriental durante las etapas extensionales. Tanto estas fallas, como la de La Peña rejugaron también durante el acortamiento alpino, en este caso como fallas inversas (Fig. 1A y 6) o con efecto de contrafuerte, probablemente con un componente de desgarre levógiro (Uzkeda et al., op. cit.). Las relaciones de corte entre los diferentes sistemas de fallas (Figs. 2A y 6) ilustran sobre la edad relativa de los mismos durante el acortamiento alpino. Las más antiguas son las de rumbo E-O (Llanera y Careses), las cuales están cortadas por las de traza NE-SO; la más moderna es la Falla de Ventaniella, de rumbo NO$\mathrm{SE}$, que trunca los dos sistemas mencionados.

Por otro lado, la Falla de la Peńa probablemente se corresponde hacia el sur con la falla de Pajares (Aller, 1986), rebautizada como retrocabalgamiento varisco de Pajares (Alonso et al., 2009b) (Fig. 7). Se trata del único retrocabalgamiento mayor de la Zona Cantábrica, lo que permite explicar el des- censo de su labio oriental en las fallas de La Peña y Villaviciosa durante la extensión mesozoica. Este retrocabalgamiento y el Cabalgamiento de León, al que se encuentra asociado, cortan estructuras previas y pueden considerarse de los más profundos del antepaís varisco; son asimismo los de mayor longitud (Alonso et al., 2009b), tienen gran importancia metalogenética (Luque Cabal y Martínez García, 1983) y han sido preferentemente reactivados durante la deformación alpina (Pulgar et al., 1999; Alonso et al, 2007) (Fig. 7).

\section{Conclusiones}

En un talud de la autovía del Cantábrico junto a la localidad de Lamasanti, la Falla de Llanera se muestra como normal en su parte inferior y como inversa en la parte superior del afloramiento, rasgo característico de las fallas normales que han sufri-

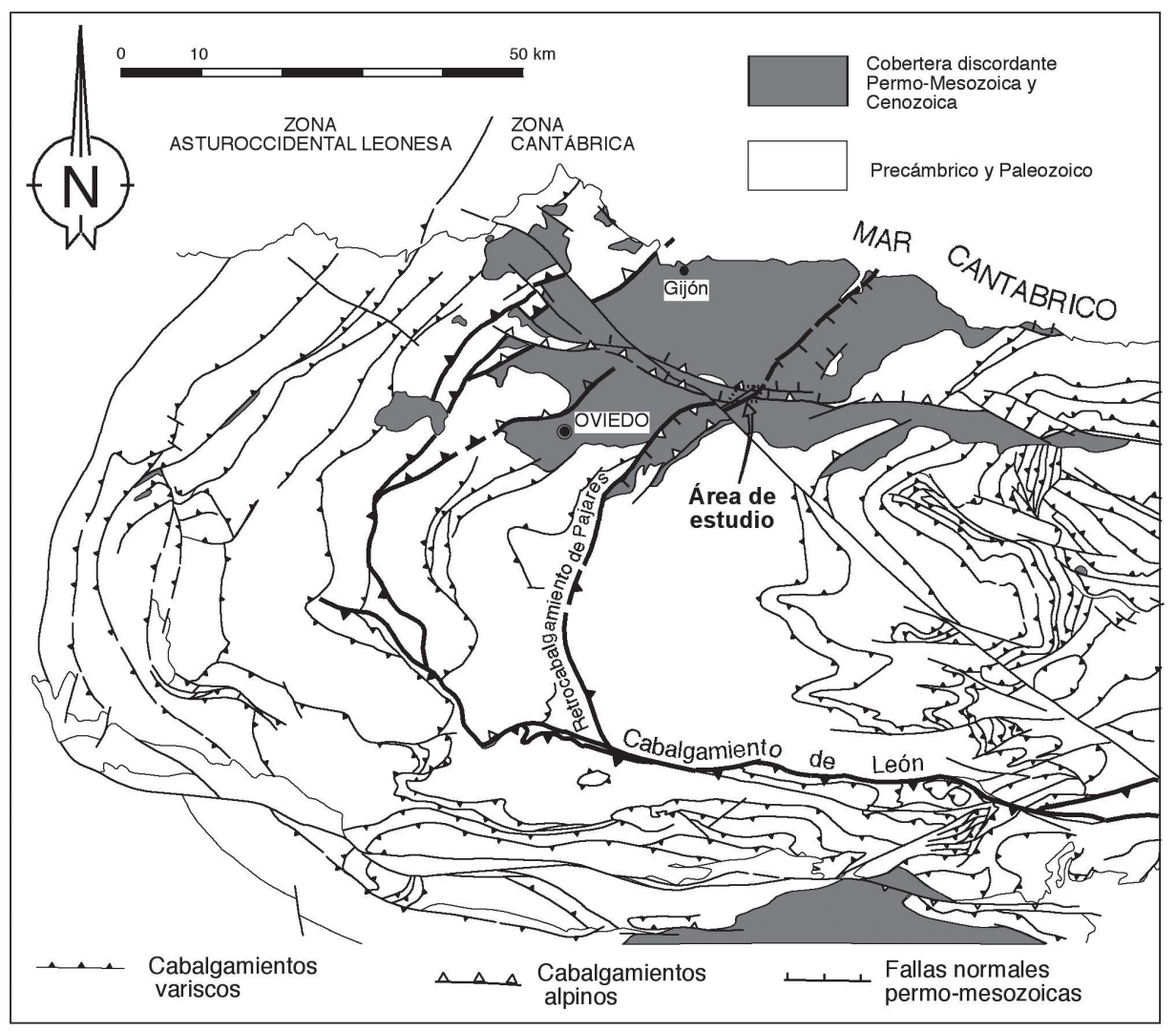

Fig. 7. Esquema estructural del sector occidental de la Zona Cantábrica resaltando (con línea negra gruesa) algunos de los principales cabalgamientos variscos reactivados durante el acortamiento alpino y/o la extensión previa que generó las cuencas permo-mesozoicas. Modificado de Alonso et al. (2009b). 
do inversión tectónica cuando el desplazamiento normal fue mayor que el inverso. El juego normal tuvo lugar durante el Jurásico Superior-Cretácico Inferior pre-Barremiense y el inverso durante la deformación alpina. En el sector que presenta juego normal la falla pone en contacto las sucesiones pre-rift de ambos labios y en la parte superior con juego inverso la sucesión post-rift está presente en el bloque inferior. En esta misma localidad se ha datado con polen la base de la secuencia post-rift en dicho bloque, obteniéndose una edad Barremiense, la cual se registra por primera vez en la cuenca mesozoica asturiana y es la edad más antigua registrada en la sucesión post-rift. Otra falla mayor del área de Sariego, denominada Falla de Careses, es paralela a la anterior y se muestra como una falla normal invertida en su sector oriental, mientras en el sector occidental del área estudiada puede interpretarse como una falla inversa de atajo (short cut) de la falla normal mencionada. Su juego inverso se refleja en el relieve actual dando lugar a un escarpe mucho más notable que el de la Falla de Llanera, a pesar de que el desplazamiento inverso es mucho mayor en esta última; ello puede indicar un movimiento más reciente de la Falla de Careses. Las dos fallas mayores mencionadas se encuentran desplazadas por fallas de rumbo SO-NE, cuya traza cartográfica y rasgos cinemáticos coinciden con las fallas de Villaviciosa y de la Peña, situadas respectivamente al norte y sur del área estudiada, y con las que se pueden correlacionar. Estas fallas de rumbo SO-NE representan la reactivación de estructuras variscas subyacentes, que tienen esa misma dirección; durante el Pérmico jugaron en extensión, controlando los espesores de dicha sucesión y durante la orogenia alpina actuaron como fallas de desgarre con ligero movimiento vertical, generando pliegues subparalelos a las mismas. Durante el acortamiento alpino, la falla de Ventaniella, con rumbo NO-SE, es la más joven de todas, ya que corta tanto las fallas E-O (Llanera y Careses) como las fallas NE-SO.

\section{Agradecimientos}

Este trabajo ha sido financiado parcialmente con los proyectos FC-15-GRUPIN14-044 y CGL201784419. Agradecemos a Jorge Gallastegui y a un revisor anónimo su contribución a la mejora del manuscrito original.

\section{Bibliografía}

Almela, A. y Ríos, J. M. (1962): Investigación del Hullero bajo los terrenos mesozoicos de la Costa Cantábrica (zona de Oviedo-Gijón-Villaviciosa-Infiesto), Empresa Nacional Adaro de Investigaciones Mineras, Madrid, $171 \mathrm{p}$.

Aller, J. (1986): La estructura del sector meridional de las unidades del Aramo y la Cuenca Carbonifera Central. Principado de Asturias, Consejería de Industria y Comercio, $180 \mathrm{p}$.

Alonso, J. L., Pulgar, J. A., García-Ramos, J. C. y BARbA, P. (1996): Tertiary basins and Alpine tectonics in the Cantabrian Mountains, en P. F. Friend y C. J. Dabrio (eds.), Tertiary Basins and of Spain: The Stratigraphic Record of Crustal Kinematics. Cambridge University Press, Cambridge, 214-227.

Alonso, J. L., Martínez Abad, I. y García-RaMos, J. C. (2007): Nota sobre la presencia de una sucesión cretácica en el Macizo de Las Ubiñas (Cordillera Cantábrica). Implicaciones tectónicas y geomorfológicas. Geogaceta, 43: 47-50.

Alonso, J. L., Gallastegui, J., García-Ramos, J. C. y Poblet, J. (2009a): Estructuras mesozoicas y cenozoicas relacionadas con la apertura y cierre parcial del Golfo de Vizcaya (Zona Cantábrica-Asturias). 6. ${ }^{\circ}$ Simposio sobre el Margen Ibérico Atlántico. Guía de Campo, Oviedo, 18 p.

Alonso, J. L., Marcos, A., y Suárez, A. (2009b): Paleogeographic inversion resulting from large out of sequence breaching thrusts: The Leon Fault (Cantabrian Zone, NW Iberia). A new picture of the external Variscan Thrust Belt in the Ibero-Armorican Arc: Geologica Acta, 7(4): 451-473.

Barrón, E., Gómez, J. J. y Goy, A. (2002): Los materiales del tránsito Triásico-Jurásico en la región de Villaviciosa (Asturias, España). Caracterización palinológica. Geogaceta, 31: 197-200.

Barrón, E., Peyrot, D., Rodríguez-López, J. P., Meléndez, N., López del Valle, R., Najarro, M., Rosales, I. y Comas-Rengifo, M. J. (2015): Palynology of Aptian and upper Albian (Lower Cre- 
taceous) amber-bearing outcrops of the southern margin of the Basque-Cantabrian basin (northern Spain). Cretaceous Research, 52(A): 292-312.

Diéguez, C., Peyrot, D. y Barrón, E. (2010): Floristic and vegetational changes in the Iberian Peninsula during Jurassic and Cretaceous. Review of Palaeobotany and Palynology, 162: 325-340.

Dubar, G. y Mouterde, R. (1957): Extension du Kimméridgien marin dans les Asturies depuis Ribadesella jusqu’à Gijón. Compte Rendu Academie des Sciences de París, Série D, 244: 99-101.

Espina, R. G., de Vicente, G. y Muñoz Martín, A. (1996): Análisis poblacional de fallas alpinas en el borde occidental de la Cuenca Vasco-Cantábrica (Cordillera Cantábrica, NO de España). Geogaceta, 20(4): 936-938.

Espina, R. G., Alonso, J. L. y Pulgar, J. A. (2004): Extensión triásica en la Cuenca Vasco-Cantábrica, en J. A. Vera (ed.), Geología de España. SGE-IGME, Madrid, 338-339.

Ferrús, B. y Santanach, P. (1994): Análisis de la fracturación en la cuenca cenozoica de As Pontes (La Coruña). Geogaceta, 15: 147-149.

García-Ramos, J. C. y Gutiérrez Claverol, M. (1995): La cobertera Mesozoico-Terciaria, en C. Aramburu y F. Bastida (eds.), Geología de Asturias. Editorial Trea, 81-94.

González-Fernández, B., Menéndez-Casares, E., Gutiérrez-Claverol, M. y García-Ramos, J. C. (2004): Litoestratigrafía del sector occidental de la cuenca cretácica de Asturias. Trabajos de Geología, 24: 43-80.

Gutiérrez Claverol, M. (1973): Sinopsis estratigráfica del Cretácico de Asturias. Boletín IDEA, suplemento de Ciencias, 18: 1-118.

Jullien, J. (1974): Recherches sur les minéralisations fluorées liées à l'évolution du bassin permo-triasique des Asturies (Espagne). These Doctoral, Université de París VI, 298 p.
Lepvrier, C. y Martínez-García, E. (1990): Fault development and stress evolution of the post-Hercynian Asturian Basin (Asturias and Cantabria, northwest Spain). Tectonophysics, 184: 345-356.

Llopis Lladó, N. (1950): Mapa Geológico de los alrededores de Oviedo. Servicio Geológico, Instituto de Estudios Asturianos, Oviedo.

Luque Cabal, C. y Martínez García, E. (1983): Análisis tectometalogenético de las mineralizaciones en el Carbonífero de la Zona Cantábrica (NW de España) En: C. R. X Congreso Internacional de Estratigrafía y Geología del Carbonifero, 3: 95-104, Madrid.

Martínez García, E., Coquel, R., Gutiérrez Claverol, M. y Quiroga, J. L. (1998): Edad del «Tramo de transición» entre el Pérmico y el Jurásico en el área de Gijón (Asturias, NW de España). Geogaceta, 24: 215-218.

Menéndez Casares, E., González Fernández, B., Alonso, J. L., García Ramos, J.C., Gutiérrez Claverol, M., Pulgar, J. A. y Aramburu-Zabala, C. (2010): Mapa Geológico de España Escala 50.000, Hoja N. ${ }^{\circ} 29$ (Oviedo). IGME, Madrid.

Merino-Tomé, O., Suárez Rodríguez, A., Alonso J. L., González Menéndez, L., Heredia, N. y Marcos, A. (2014): Mapa Geológico Digital continuo (GEODE) E. 1:50.000, Principado de Asturias (Zonas: 1100-1000-1600). IGME. Disponible en: http://cuarzo.igme.es/sigeco/default.htm

Najarro, M., Rosales, I., Moreno-Bedmar, J. A., de Gea, G.A., Barrón, E., Company, M. y DelaNOY, G. (2011): High-resolution chemo- and biostratigraphic records of the Early Aptian oceanic anoxic event in Cantabria (N Spain): Palaeoceanographic and palaeoclimatic implications. Palaeogeography, $\mathrm{Pa}$ laeoclimatology, Palaeoecology, 299: 137-158.

Navarro Vázquez, D., Leyva Cabello, F., Rodríguez Fernández, L. R., Martínez Cienfuegos, F., Villa Otero, E., Horvath Mardones, V. y Heredia Carballo, N. (1988): Cuencas carboníferas ocultas por la cobertera mesozoico-terciaria en Asturias. IGME, Madrid, $100 \mathrm{p}$. 
Peyrot, D., Rodríguez-López, J. P., Barrón, E. y MelÉndez, N. (2007): Palynology and biostratigraphy of the Escucha Formation in the Early Cretaceous Oliete Sub-basin, Teruel, Spain. Revista Española de Micropaleontología, 39(1-2): 135-154.

Pieren, A. P., Areces, J. L., Toraño, J. y Martínez GARCÍA, E. (1995): Estratigrafía y estructura de los materiales permotriásicos del sector Gijón-La Collada (Asturias). Cuadernos de Geología Iberica, 19: 309-335.

Pulgar, J. A., Alonso, J. L., Espina, R. G., y MaRín, J. A. (1999): La deformación alpina en el basamento varisco de la Zona Cantábrica. Trabajos de Geología, 21: 283-294.

Quintana, L., Alonso, J. L., Pulgar, J. A. y RoDRÍGUEZ-FERnÁNDEZ, L. R. (2006): Transpressional inversion in an extensional transfer zone (the Saltacaballos fault, northern Spain). Journal of Structural Geology, 28: 2038-2048.

Ramírez del Pozo, J. (1969): Bioestratigrafía y paleogeografía del Jurásico de la costa asturiana (zona de Oviedo-Gijón-Villaviciosa). Boletín Geológico y Minero, 80(4): 307-332.

Sánchez de la Torre, L., Águeda Villa, J. A., Colmenero, J. R. y Manjón, M. (1977): La serie permotriásica en la región de Villaviciosa (Asturias). Cuadernos de Geología Ibérica, 4: 329-338.

Schroeder, R. y Wienands, A. K. (1966): Über die marine Unter- kreide der asturischen Küste (Nord-Spanien). Neues Jahrbuch für Geologie und Paläontologie, 7: 425-433.

Suárez Rodríguez, A. (1988): Estructura del área Villaviciosa-Libardón (Asturias, Cordillera Cantábrica). Trabajos de Geología, 17: 87-98.

Suárez Vega, L. C. (1974): Estratigrafía del Jurásico en Asturias. Cuadernos de Geología Ibérica, 3(1): $1-368$.

Tejerina Lobo, L. y Vargas Alonso, A. (1980): Descripción geológica del distrito minero de La Collada (Fluorita teletermal, Asturias). Tecniterrae, 6 (35): 44-53.
Traverse, A. 2007: Paleopalynology, 2nd edition. Springer, Dordrecht, The Netherlands. 813 p.

TRInCÃo, P. R. (1990): Esporos e granos de polen do Cretácico Inferior (Berriasiano-Aptiano) de Portugal: Paleontologia e Biostratigrafia. Tesis Doctoral, Universidade Nova Lisboa, Portugal.

Truyols, J., García-Ramos, J. C., Casanovas-Cladellas, M. L. y Santafé-Llopis, J. V. (1991): El Terciario de los alrededores de Oviedo. Acta Geológica Hispánica, 26(3-4): 229-233.

Uzkeda, H., Bulnes, M., Poblet, J., García-Ramos, J. C. y Piñuela, L. (2013): Buttressing and reverse reactivation of a normal fault in the Jurassic rocks of the Asturian Basin, NW Iberian Peninsula. Tectonophysics, 599: 117-134.

Uzkeda, H., Bulnes, M., Poblet, J., García-Ramos, J. C. y Piñuela, L. (2016): Jurassic extension and Cenozoic inversion tectonics in the Asturian Basin, NW Iberian Peninsula: 3D structural model and kinematic evolution. Journal of Structural Geology, 90: 157-176.

Valenzuela, M., García-Ramos, J. C. y Suárez de Centi, C. (1986): The Jurassic sedimentation in Asturias (N Spain). Trabajos de Geología, 16: 121-132.

Villanueva-Amadoz, U., Pons, D., Díez, J.-B., Ferrer, J. y Sender, L.M. (2010): Angiosperm pollen grains of Sant Just site (Escucha Formation) from the Albian of the Iberian Range (north-eastern Spain). Review of Palaeobotany and Palynology, 162(3): 362-381.

Wagner, R. y Martínez García, E. (1982): Description of an early Permian flora from Asturias and comments on similar ocurrences in the Iberian Peninsula. Trabajos de Geologia, 12: 273-287.

Williams, G. D., Powell, C. M. y Cooper, M. A. (1989): Geometry and kinematics of inversión tectonics. In: M. A. Cooper y G. D. Williams (eds.), Inversion Tectonics. Geological Society Special Publication, 44: 3-15. 